Original article

\title{
Resilience trajectories to full recovery in first-episode schizophrenia
}

\author{
Anne-Kari Torgalsbøen ${ }^{\mathrm{a}, *}$, Susie $\mathrm{Fu}^{\mathrm{a}, \mathrm{b}}$, Nikolai Czajkowski ${ }^{\mathrm{a}}$ \\ a Department of Psychology, University of Oslo, Norway \\ ${ }^{\mathrm{b}}$ Vestre Viken Hospital Trust, Norway
}

\section{A R T I C L E I N F O}

\section{Article history:}

Received 16 March 2018

Received in revised form 26 April 2018

Accepted 27 April 2018

Available online 3 May 2018

\section{Keywords:}

Course

Longitudinal

Recovery

Medication free

Psychosis

Functional outcome

\begin{abstract}
A B S T R A C T
Background: Resilience is successful adaptation despite adversity. This personality trait has the potential to add new knowledge to how to achieve a successful outcome, but resilience has been understudied in schizophrenia. The objective of the present study is to investigate if there are significant differences in resilience development among fully recovered and non-recovered patients with first episode schizophrenia (FES).

Methods: In the ongoing Oslo Schizophrenia Recovery Study spanning 10 years, 28 first-episode patients are interviewed and assessed yearly with comprehensive criteria of full recovery, a measure of social and role functioning and resilience, the Connor-Davidson Resilience Scale. The present study includes data from six follow-ups over four years. Working or studying, having symptoms that are stably mild or absent for two years or more, having contact with friends and/or dating, participating in leisure activities and living independently define full recovery.

Results: At the four-year follow-up, 55\% were sustained full/partly recovered. Ten percent of those fully recovered were no longer in treatment. Choosing the overall best linear mixed model, we found a significantly larger increase in resilience score among the fully recovered than among those not recovered.

Conclusions: Based on the theoretical rationale that resilience is activated differently in persons who experience adversity, the significant increase in resilience in the fully recovered group indicates that this psychological trait is present to a higher degree in fully recovered. These results highlight resilience as a factor associated with increased recovery in FES adding to the small literature on improvement among these patients and thus have important clinical implications.
\end{abstract}

(C) 2018 Elsevier Masson SAS. All rights reserved.

\section{Introduction}

As early as 1970, Garmezy [1] reported on two kinds of patients with schizophrenia: process and reactive. Chronic courses characterized the process patients, whereas reactive patients had courses with a return to a functional life after their psychotic breakdown, thereby illustrating a positive adaptation to the illness. Resilience was not part of the descriptive picture of these atypical cases, but the psychosocial development of these patients was an early example of signs of premorbid resilience indicators [2]. The subset of patients who evidenced recovery and adaptive patterns have been considered to be somewhat atypical, and have been afforded little attention. With some exceptions [3-8] there has been a lack of positively framed research in schizophrenia, and very little research has been conducted into how a person with

\footnotetext{
* Corresponding author at: Department of Psychology, University of Oslo, P.O. Box 1094 Blindern NO-0317 Oslo, Norway.

E-mail address: a.k.torgalsboen@psykologi.uio.no (A.-K. Torgalsbøen).
}

schizophrenia arrives at a successful outcome. In this perspective, one might argue that it is a limitation of research, rather than limitations on the ability of individuals, that recovery has not been more widely explored and acknowledged. Recovery in schizophrenia is considered an outcome measure, a process and a definition [9] leading to conceptions of recovery giving equal weight and importance to clinical and personal recovery for the assessment of the final functional outcome.

Although recovery rates for people with first-episode schizophrenia (FES) are still somewhat uncertain due to a wide variety of outcome definitions used [10] as well as lack of consensus on the definition of recovery, a recent meta-analysis of long-term outcome studies concluded that remission and recovery rates in this group may be more favourable than previously thought [11]. The pooled prevalence of recovery among 9642 individuals with first-episode psychosis was 38\% (35 studies, mean follow-up 7.2 years) [11]. To increase the knowledge of how persons facing such a profound adversity as schizophrenia successfully recover, we must turn to the field of developmental psychopathology [12]. The recognition of the diversity of developmental outcomes and the 
complexity of developmental pathways has given impetus to the growing interest regarding the presence of successful adaptation despite adversity, the construct of resilience [13]. Resilience is somewhat different than other personality traits because it manifests in response to adversity [11] and according to Bonanno, events are not traumatic until we experience them as traumatic. [14]. Previous follow-up studies on full recovery in schizophrenia using operational consensus-based criteria show that resilience plays a significant role in sustained full recovery $[4,8]$. Some longterm studies have used resilience as describing personal resources, though without defining the concept $[15,16]$. Lately some crosssectional studies has addressed resilience in schizophrenia patients [17,18]. At present there is limited knowledge about how successful outcomes in first-episode schizophrenia (FES) are achieved as well as which factors contribute to recovery.

To fill this knowledge gap we have to investigate the development of resilience in persons who are fully recovered. Secondly, we have to define resilience according to how it is conceptualized in the field of developmental psychopathology where resilience embodies the personality traits that enable one to thrive in the face of adversity, to bounce back from difficult experiences [19]. The instrument that in our opinion measures resilience according to this definition is a scale developed by Connor and Davidson [20], the Connor and Davidson Resilience Scale (CD-RISC). This instrument also measures optimism and hope, which are individual traits characterizing resilient individuals $[21,22]$.

To the best of our knowledge, the Oslo Schizophrenia Recovery study is the first prospective study using the most comprehensive and strict definition of full recovery ([23] in a year-by-year -assessment as part of a modern longitudinal research design to investigate the rate of full recovery in FES. Moreover, we identify the time point during the follow-up period when individuals meet criteria for remission and full recovery, as well as tracking the development of resilience. The aim of the present study is to investigate the relationship between resilience defined as a personality trait and full recovery in FES, and more specifically, if there are significantly different resilience developments among fully recovered and non-recovered groups.

\section{Methods and measures}

\subsection{Design}

The Oslo schizophrenia recovery study [24-26] has a prospective longitudinal design, with 12 follow-up points spanning a period of 10 years. This design makes it possible to assess full and sustained recovery, as well as studying resilience over time in a sample not confined to the relapsing patients most often seen in hospital/inpatient settings. It also allows for a testing of causal relationships. The study is ongoing, and here we present the results from the four-year follow-up. During this time, all participants were assessed at six time points, enabling us to capture both the fluctuations and stability in recovery and resilience.

\subsection{Participants}

Over a period of four years (2007-2011), 31 patients with firstepisode schizophrenia were referred to the study. They were recruited from mental health service institutions in the Oslo area, and shortly after admittance, their treating clinicians referred them to the project. Twenty-eight out of 31 patients fulfilled the following inclusion criteria: age $\geq 18$ years, the first episode of mental illness was within the spectrum of schizophrenia and psychosis according to DSM-IV [27] and referral within five months of their first contact with the mental health service institutions. Exclusion criteria were affective disorders, IQ $<70$ and head trauma, and that all patients could read and write fluent Norwegian. The mean age of the included subjects at baseline was 21.0 years (S.D.2.6) with a mean of 12.7 years of education. $21.4 \%$ was diagnosed with schizoaffective disorder, the majority with schizophrenia. The total symptom level was 80.8 (SD 16.5) on the Positive and Negative Syndrome Scale (PANNS) [28], while the positive symptom level was 18.5 (SD 5.6) and the negative symptom score was 21.5 (SD 3.8). Regarding illegal substances, $64.3 \%$ had previously used cannabis and amphetamines, but at the time of inclusion, only $3.6 \%$ had substance abuse (mainly recreational drugs). Moreover, 57\% were hospitalized and $43 \%$ were outpatients at baseline.

In the follow-up period, the majority of patients was provided with treatment by their local mental health service institution, through antipsychotic medication, primarily second-generation antipsychotics, in addition to psychoeducation and/or CBT and case management. The retention rate at 4-year follow- up is high (78.6\%). All patients were retained during the first three followups, while three patients left the study during the 2-year follow up and an additional three participants dropped out during the 3-year follow-up.

The reasons for dropout were mainly refusal to participate due to anxiety, a lack of insight into having a mental illness, finding participation in research not useful and non-response to attempt at contact. On every measurement occasion, the patients completed the assessments described below. After carefully describing the study and the procedures involved, written informed consent was obtained from all participants, and the Regional Committee for Research Ethics (REK) approved the study.

\subsection{Clinical instruments}

The clinical interviews and tests of the participants were done within the first five months of their admission to hospitals or outpatient clinics, and were carried out by an experienced clinical psychologist. Diagnoses were established using the Structural Clinical Instrument of Diagnosis for DSM-IV Axis I disorders (SCID-I), modules A-D. The degree of symptoms and psychopathology was measured with the Positive and Negative Syndrome Scale (PANSS) [28]. Furthermore, a semi-structured interview was used, and based on this information level of social and role functioning, was measured by the Global Functioning: Social (GF: Social) and the Global Functioning: Role (GF: Role) [29]. These two measures of social and role functioning disentangle social- from role-functioning domains, detect changes in functioning over time, and provide brief and easy-to-use clinician ratings, while taking age and phase of illness into account.

In this instrument, the social scale assesses the quantity and quality of peer relationships, level of peer conflict, age-appropriate intimate relationships and involvement with family members. The role scale refers to performance in school, at work or as a homemaker. We consider these measures as suitable for prospectively following first-episode individuals.

To assess resilience, the Connor-Davidson Resilience Scale (CDRISC) was chosen. This scale was translated into Norwegian and demonstrated sound psychometric properties, and also distinguishes between persons with a greater and lesser resilience [20]. The scale is comprised of 25 items, each rated on a 5-point Likert scale (0-4), with higher scores reflecting a greater resilience. It includes items such as for example: "I tend to bounce back after illness or hardship" and "I have close and secure relationships".

\subsubsection{Symptom remission}

The symptom remission criteria for schizophrenia (24) are based on an evaluation of eight groups of symptoms of the PANSS: P1 
(delusions), G9 (unusual thought content), P3 (hallucinatory behavior), P2 (conceptual disorganization), G5 (mannerisms and posturing), $\mathrm{N} 1$ (blunted affect), N4 (social and emotional withdrawal) and N6 (lack of spontaneity). The score on these items must be mild or less $(<3)$, using the 1-7 range for each item, with a duration of six months as a minimum threshold. A score of six (moderate impairment or above) on the GF: Social and Role is required to be considered in remission.

\subsubsection{Full recovery}

The full recovery criteria used is a combination of Andreasen and collaborators [30], with the operational recovery criteria developed by Liberman and collaborators [23]. The remission criteria are based on the evaluation of the eight groups of symptoms of the PANSS. The score on these items must be mild or less $(<3)$, with a duration of two years. In addition, the subject must fulfill the following criteria concerning psychosocial functioning: at least part-time work or school, living independently of supervision by family and at least once weekly socializing with peers or otherwise involved in recreational activities that are ageappropriate and independent of professional supervision. To be considered fully recovered, a score of eight (adequate social/ interpersonal functioning and good role functioning) on the GF: Social and Role is required. It is important to recognize that not all members of the public without mental disorders would meet the level of role and social functioning required by our criteria. As a result, if not leading to significant impairment in social and role functioning, it makes sense to allow minor impairments (in housing, work or intimate relationships), and define these subjects as partly recovered. Thus, to be considered partly recovered, the remission criteria must be fulfilled for a duration of at least two years, with an exception for one of the psychosocial criteria.

We undertook an inter-rater reliability assessment in order to establish accuracy of remission and recovery in schizophrenia according to the full recovery criteria. Thirty-six clinical protocols were rated by an independent rater, three for each patient fulfilling the criteria for either full or partial recovery at the current time points showing an inter-rater reliability of 0.69 (Cohen's kappa), which is a good inter-rater agreement.

\subsection{Data analyses}

To investigate how resilience developed over the four-year follow-up period, we fitted a series of longitudinal growth curve models (linear mixed models) in SPSS 20.0 [31]. Multilevel modelling is a flexible statistical approach that can accommodate repeated observations with missing entries [32]. All models were fitted using full information maximum likelihood, and an unstructured covariance matrix for the random effects.
The baseline model to which the subsequent models were compared contained only a fixed and random intercept. Model two allowed for a linear change in resilience over time, whereas Model 3 allowed the linear resilience trajectory to vary across groups defined by full recovery at T6 (four years) by including a time $\mathrm{x}$ group interaction term. The overall best model was chosen based on the lowest AIC information criterion [33].

\section{Results}

\subsection{Remission status}

A stacked bar chart indicating the number of participants in different categories of remission and recovery is given in Fig. 1:

At the four-year follow-up, $29 \%$ of the subjects were in remission and $55 \%$ were sustained full/partly recovered. For those who had sustained their recovery, the duration ranged from one to four years. An additional finding was that among the fully recovered, six subjects were not on antipsychotic medication at follow-up, and the majority had not been taking antipsychotic medication in the three preceding years. As early as the second year of follow-up, participants achieve full recovery. The rates of full recovery and sustained full recovery are steadily increasing over the four-year follow-up period.

As required by the criteria for full/partial recovery, Figs. 2 and 3 illustrate that the improvement in social and role functioning in the same follow-up period is particularly evident among those who attain a full/partial recovery at the four-year follow-up (right panel).

\subsection{Longitudinal modelling}

Results from the linear mixed models are given in Table 1:

Estimates in the baseline Model 1 suggest that there are significant differences in resilience across participants, when the effect of time is disregarded ( $p=0.018)$. Model 2 includes a linear effect of time which was found to be a statistically significant predictor of resilience $(p=0.001)$. Model 3 allowed for the effect of time to be moderated by recovery status at the four-year follow-up (T6), thus predicting different developmental trajectories in resilience within the two groups (recovered vs. non-recovered). Results indicate that resilience in participants not recovered at t6 displayed a small increase of 1.38 units per year, although this increase was not statistically significant $(p=0.167)$. The resilience among participants recovered at $\mathrm{t} 6$ was found to increase significantly more steeply than among those non-recovered, as the interaction parameter contributed an additional 3.4 unit increase in resilience per year $(\mathrm{p}=0.026)$. The baseline differences
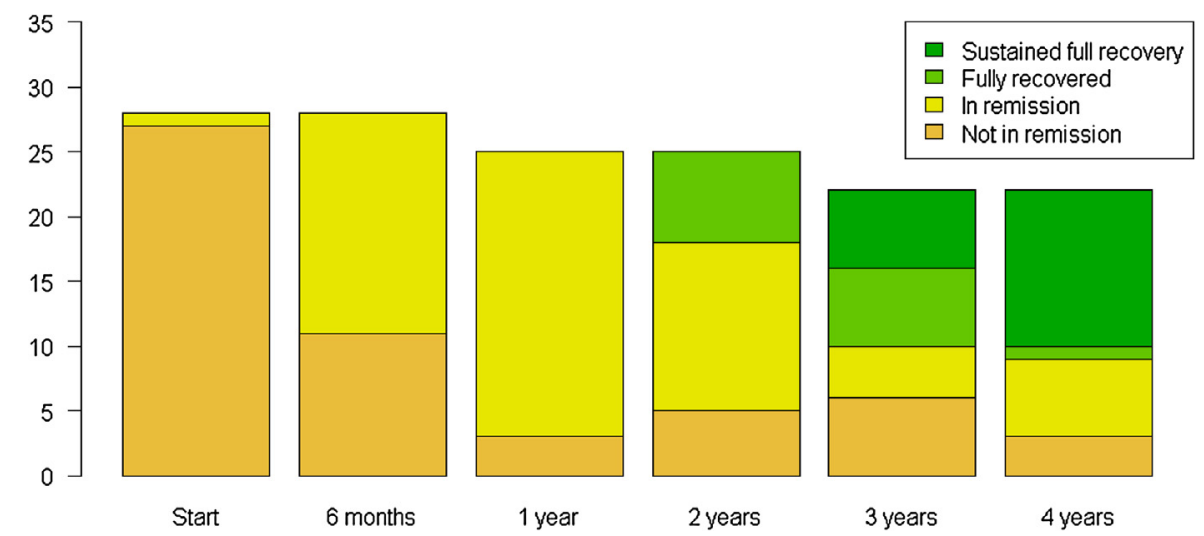

Fig. 1. Number of participants meeting the criteria for the four levels of recovery across six measurement waves. 


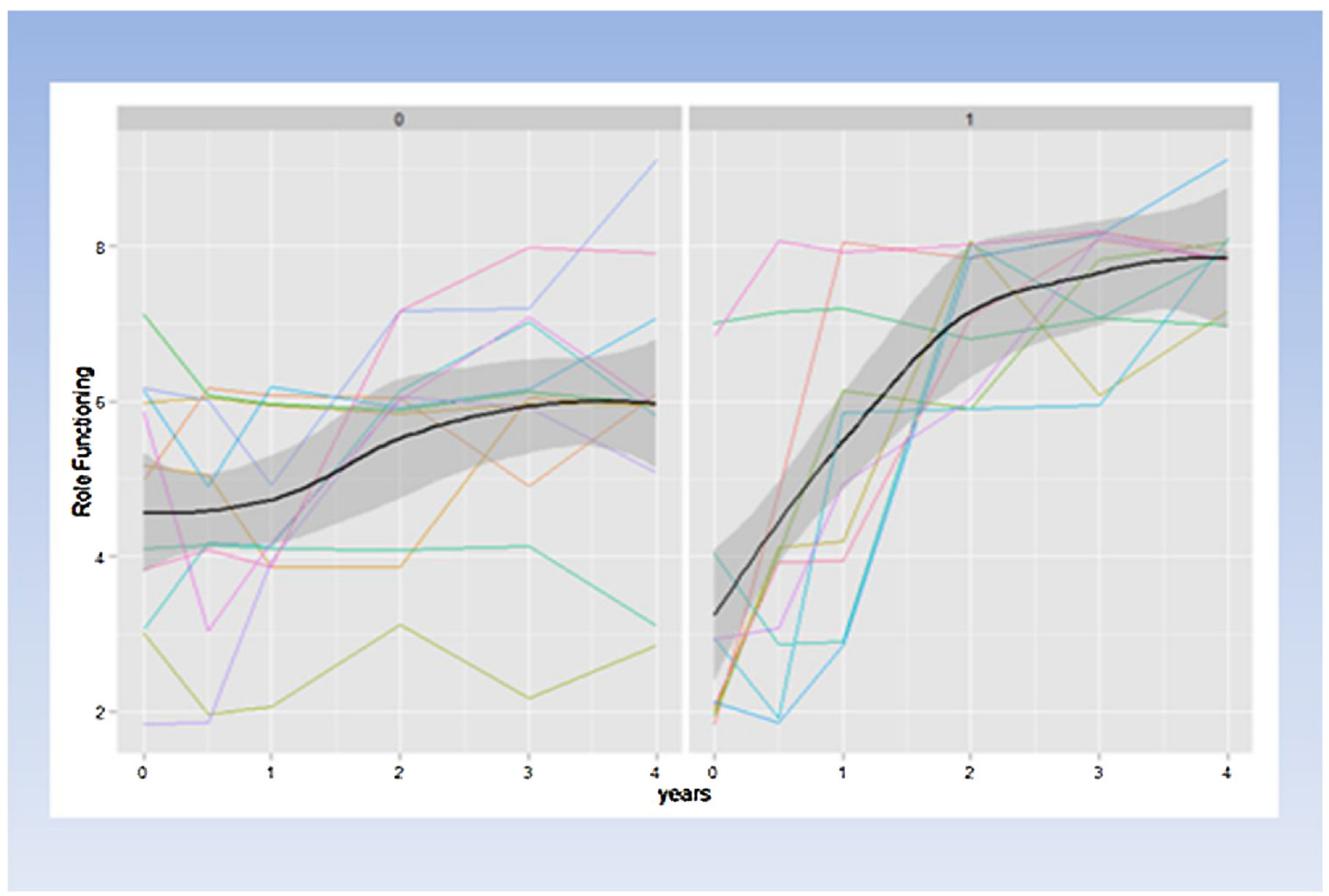

Fig. 2. Individual and mean (bold) development in role functioning for individuals fully recovered at four-year follow up (right) and not recovered (left).

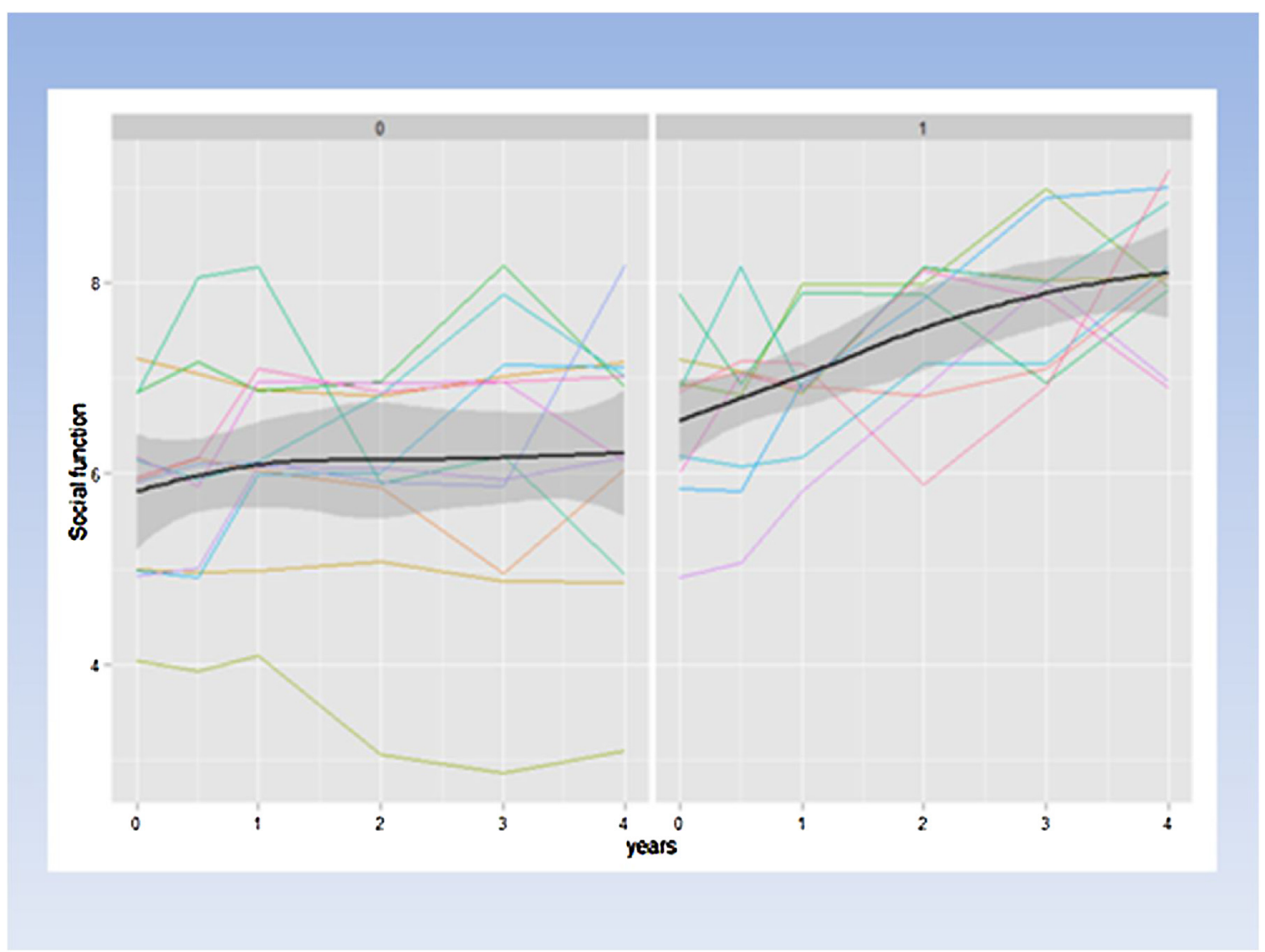

Fig. 3. Individual and mean (bold) development in social functioning for individuals fully recovered at four-year follow up (right) and not recovered (left). 
Table 1

Results from growth curve models.

\begin{tabular}{|c|c|c|c|}
\hline & $\begin{array}{l}\text { Model } 1 \\
\text { Est. }\end{array}$ & $\begin{array}{l}\text { Model } 2 \\
\text { Est. }\end{array}$ & $\begin{array}{l}\text { Model } 3 \\
\text { Est. }\end{array}$ \\
\hline \multicolumn{4}{|l|}{ Fixed effects } \\
\hline Intercept & $66.981^{* *}$ & $61.837^{* *}$ & $61.728^{* *}$ \\
\hline Recovery $_{6}$ & & $2.919^{* *}$ & -0.130 \\
\hline Time & & & 1.380 \\
\hline Time $\mathrm{x}$ Recovery $\mathrm{t}_{\mathrm{t}}$ & & & $3.422^{*}$ \\
\hline \multicolumn{4}{|l|}{ Random effects } \\
\hline Residual & $118.187^{* *}$ & $80.189 * *$ & $80.183^{* *}$ \\
\hline Intercept & $51.453^{*}$ & $57.163^{*}$ & $57.928^{*}$ \\
\hline Time & & 7.233 & 4.237 \\
\hline \multicolumn{4}{|l|}{ Model fit indices } \\
\hline$-2 \mathrm{LL}$ & 1017.317 & 990.357 & 983.143 \\
\hline AIC & 1023.317 & 1002.357 & 999.143 \\
\hline
\end{tabular}

in resilience predicted by Model 3 were not found to be significant $(p=0.975)$. Among the models tested, we selected Model 3 as the best fitting based on the lowest AIC level (999.143).

\section{Discussion}

At the four-year follow-up, approximately 55\% of the sample of patients met the comprehensive criteria for a full/partial recovery. Thus, we confirmed the positive early trajectory for the FES reported earlier from this study [24-26] and the results are in line with other studies finding that, a substantial proportion with first-episode psychosis do recover. Schizophrenia represents a major adversity in a young person's life, a stressor that can be so intense that the resilience can be overwhelmed. This might explain the no-difference baseline results in resilience in this sample. The results may indicate that resilience is negatively affected in the acute phase of illness, but once the disease is stabilized through treatment individual differences in resilience will emerge and contribute differently to the person's functioning and recovery process. It is only when you are with obstacles, stress and other environmental threats that resilience, or the lack of it, emerges [14].

Sixteen\% of the subjects in the current study achieve full recovery by year 2 and sustain their recovery in year 4, thereby showing stability in their recovery. Using comparable recovery criteria, results from the OPUS study [34](mean age 26.0) showed that $15.7 \%$ of the sample were recovered after five years, but these did not belong to the same group that was recovered at two-year follow-up, which showed a recovery rate of $17 \%$. The design of the current study enables us to study the recovery process in detail in the same subjects, hence allowing us to capture changes or stability between both the assessment points and a sustained full recovery. It is further important to underscore that the most comprehensive and strict definition of full recovery is used in a year-by-year assessment that comes much closer to reality [35] than current multi-follow-up studies, including assessment points many years apart, thereby being less sensitive to changes that occur in-between the assessment points(for instance [34,36]). Three or four cross-sectionals used in many studies "do not a longitudinal make" [35].

The identification of resilience courses toward full recovery also explains some of the heterogeneity in trajectories in FES, showing that a group of patients early after the acute phase (year 2) function adequately, and are significantly increasing their resilience and sustaining their recovery. These results are in line with recent meta-analysis [11], indicating that the course of illness is not progressive for the majority of patients.

These findings and findings from long-term follow-ups on older samples of sustained fully recovered schizophrenia patients $([4,8]$ show that resilience is at play, both in the early and late course of illness. The fact that approximately $10 \%$ of those fully recovered at year 4 were not on antipsychotic medication, and were no longer in treatment substantiate the impact of resilience. These subjects who are well and no longer in contact with mental health-services may be disproportionately lost to follow-up, thus probably contributing to an underestimation of the recovery rate [11]. Harrow and Jobe [37] found a subgroup of $20-35 \%$ of patients with schizophrenia, who after showing signs of better functioning and partial or global recovery for a period of time, have gone off or been taken off medication. Our study, consistent with accumulating evidence [38-40], show that a group of FES patients can improve and sustain their recovery without antipsychotic treatment, even in the early course of illness.

The greatest improvement in social and role functioning among those who are fully recovered in year 4 seems to take place during the first two years of treatment, following to a large degree the same pattern as the development of resilience. The first years therefore seems to represent a "window of opportunity" for recovery, making it possible to differentiate between early and late recoveries.

These results show how persons with FES can arrive at positive outcomes by demonstrating a strong increase in function and resilience score following the recovery. Whether the significant increase in resilience among the fully recovered contributes to improvement, or is a result of their improvements, remains an open question since it is difficult to conclude on causal direction.

Other contributing factors to the different development in resilience among the fully recovered and non-recovered could be that the latter have experienced more chronic stress due to a longer period of mental illness. In addition to the intensity of the stressor represented by the psychosis, the duration of the stressor might have a repeated and cumulative impact on resources and adaptation over time. Early treatment, participating in a positively framed research project as this one, as well as the changes in social attitudes and outlooks for persons with first-episode schizophrenia in Norway, are all protective environmental factors that may have contributed to the positive outcome of the participants in the present study.

The identification of sustained full recovery in the early course as well as different resilience courses has important clinical implications. Based on the premise that FES patients will respond differently to the adversity schizophrenia represents, the identification of both high-resilience patients and the highly vulnerable with less resilience might enable clinicians to differentiate and personalize treatment in a better way. Fully recovered patients seems to conceptualize the adversity of psychosis as a chance to learn and grow, perhaps framing this experience differently than the non- recovered. By building personal resilience, clinicians can contribute to an increased benefit from treatment, especially among less resilient patients. Reducing vulnerability could be done by providing treatments focusing on meaning-making [41] and coping abilities, thus improving competence among less resilient patients in order to reduce the likelihood of future episodes.

Resilience building strategies such as savoring and mindfulness, gratitude, practicing acts of kindness and humor are novel and have rarely been used to treat people with serious mental illness [42]. All these strategies focus on increasing positive emotions and well-being, rather than remediating weaknesses or the ability to manage stress. According to Cohn et al. [43] short- term effects of positive emotions lead to long-term physical, social and psychological resources, including resilience.

\subsection{Limitations}

The small sample size limit the generalizability of our results. However, a large sample may be hard to attain for longitudinal 
studies with many repeated measurements. Furthermore, the sample seems to be representative for first -episode patients since it represents about $60 \%$ of the incidence cases from the catchment area. Another potential limitation is that we cannot rule out the possibility that the well-functioning and favorable outcome of those with sustained full recovery has influenced their attitudes and in this way influenced scores on the resilience scale. The study's strengths are the prospective longitudinal design with yearly assessments, the use of a comprehensive definition of full recovery, sound psychometric measures of resilience and functional outcome and the high retention rate, including fully recovered subjects no longer in treatment who often drop out of longitudinal studies.

\subsection{Conclusions}

This is the first longitudinal study of FES showing a sustained full recovery early in the course and significant differences in the development of resilience among those who achieve full recovery at four-year follow-up and those who did not. Ten\% of those fully recovered were not on antipsychotic medication, and were no longer in treatment. These subjects who are well and no longer in contact with mental health-services may be disproportionately lost to follow-up, thus probably contributing to an underestimation of the recovery rate in FES [11]. Replication of the results is needed with larger sample size.

\section{Role of the funding source}

The Department of Psychology, University of Oslo funded the study. This funding source has no role in the design of this study, nor during its execution, analyses, interpretation of the data, and decision to submit results.

\section{Disclosure of interest}

All authors declare that they have no competing interest.

\section{Acknowledgement}

The authors gratefully acknowledge the contribution of the participants to this study.

\section{References}

[1] Garmezy N.. Process and reactive schizophrenia: some conceptions and issues Schizophr Bull 1970;1(2):30-74.

[2] Cicchetti D, Garmezy N. Prospects and promises in the study of resilience. Dev Psychopathol 1993;5(4):497-502.

[3] Harding CM, Brooks GW, Ashikaga T, Strauss JS, Breier A. The Vermont longitudinal study of persons with severe mental illness, II: long-term outcome of subjects who retrospectively met DSM-III criteria for schizophrenia. Am J Psychiatry 1987;144(6):727-35.

[4] Torgalsbøen A-K, Rund BR. Maintenance of recovery from schizophrenia at 20 year follow-up: what happened? Psychiatry: Interpers Biol Process 2010;73 (1):70-83.

[5] Torgalsbøen A-K, Rund BR. Lessons learned from three studies of recovery from schizophrenia. Int Rev Psychiatry 2002;14(4):312-7.

[6] Andresen R, Oades L, Caputi P. The experience of recovery from schizophrenia: towards an empirically validated stage model. Aust N Z J Psychiatry 2003;37 (5):586-94

[7] Silverstein SM, Bellack AS. A scientific agenda for the concept of recovery as it applies to schizophrenia. Clin Psychol Rev 2008;28(7):1108-24.

[8] Torgalsbøen A-K. Sustaining full recovery in schizophrenia after 15 years: does resilience matter? Clin Schizophr Related Psychoses 2012:5(4):193-200.

[9] Torgalsbøen A-K. What is recovery in schizophrenia? In: Davidson L, Harding C, Spaniol L, editors. Recovery from severe mental illnesses: Research evidence and implication for practice. 1. Boston: Boston University; 2005.

[10] Menezes N, Arenovich T,Zipursky R. A systematic review of longitudinal outcome studies of first-episode psychosis. Psychol Med 2006;36(10):1349-62.

[11] Lally J, Ajnakina O, Stubbs B, Cullinane M, Murphy KC, Gaughran F, et al Remission and recovery from first-episode psychosis in adults: systematic review and meta-analysis of long-term outcome studies. Br J Psychiatry 2017: 211(6):350-8.

[12] Masten A. Ordinary magic. Resilience processes in development. Am Psychol 2001:56(3):227-38.

[13] Sroufe LA, Rutter M. The domain of developmental psychopathology. Child Dev 1984;55(1):17-29.

[14] Bonanno GA. Uses and abuses of the resilience construct: loss, trauma, and health-related adversities. Soc Sci Med 2012;74(5):753-6.

[15] Tait L, Birchwood M, Trower P. Adapting to the challenge of psychosis: personal resilience and the use of sealing-over (avoidant) coping strategies. $\mathrm{Br} \mathrm{J}$ Psychiatry 2004:185(5):410-5.

[16] Harrow M, Grossman LS, Jobe TH, Herbener ES. Do patients with schizophrenia ever show periods of recovery? a 15-year multi-follow-up study. Schizophr Bull 2005;31(3):723-34.

[17] Galderisi S, Rossi A, Rocca P, Bertolino A, Mucci A, Bucci P, et al. The influence of illness-related variables, personal resources and context-related factors on real-life functioning of people with schizophrenia. World Psychiatry 2014;13 (3):275-87.

[18] Hofer A, Mizuno Y, Frajo-Apor B, Kemmler G, Suzuki T, Pardeller S, et al. Resilience, internalized stigma, self-esteem, and hopelessness among people with schizophrenia: cultural comparison in Austria and Japan. Schizophr Res 2016:171(1):86-91.

[19] Luthar S. Resilience in development. A synthesis of research across five decades. In: Cicchetti DCD, editor. Developmental psychopathology: risk, disorder and adaptation, 3. New York: Wiley; 2002. p. 739-95.

[20] Connor KM, Davidson JRT. Development of a new resilience scale: the connorDavidson resilience scale (CD-RISC). Depress Anxiety 2003;18(2):76-82.

[21] Seligman M. Learned optimism. New York: Random House; 1990.

[22] Taylor SE. Positive illusions. Creative self-deception and the healthy mind. New York: Basic Books; 1989.

[23] Liberman RP, Kopelowicz A, Ventura J, Gutkind D. Operational criteria and factors related to recovery from schizophrenia. Int Rev Psychiatry 2002;14 (4):256-72.

[24] Torgalsbøen A-K, Mohn C, Rishovd Rund B. Neurocognitive predictors of remission of symptoms and social and role functioning in the early course of first-episode schizophrenia. Psychiatry Res 2014;216(1):1-5.

[25] Torgalsbøen A-K, Mohn C, Czajkowski N, Rund BR. Relationship between neurocognition and functional recovery in first-episode schizophrenia: results from the second year of the Oslo multi-follow-up study. Psychiatry Res 2015;227(2):185-91.

[26] Fu S, Czajkowski N, Rund BR, Torgalsbøen A-K. The relationship between level of cognitive impairments and functional outcome trajectories in first-episode schizophrenia. Schizophr Res 2017:190:144-9.

[27] AmericanPsychiatricAssociation. Diagnostic and Statistical Manual of Mental Disorders. Washington DC: American Psychiatric Association; 1994.

[28] Kay SR, Fiszbein A, Opler LA. The positive and negative syndrome scale (PANSS) for schizophrenia. Schizophr Bull 1987;13(2):261-76.

[29] Cornblatt BA, Auther AM, Niendam T, Smith CW, Zinberg J, Bearden CE, et al. Preliminary findings for two new measures of social and role functioning in the prodromal phase of schizophrenia. Schizophr Bull 2007;33(3):688-702

[30] Andreasen N. Remission in schizophrenia: proposed criteria and rationale for consensus. Am J Psychiatry 2005;162:441-9.

[31] IBM Corp. IBM SPSS Statistics for Windows, Version 20.0. New York: IBM Corp; 2011.

[32] Jackson HJ. Reporting results of latent growth modeling and multilevel modeling analyses: some recommendations for rehabilitation psychology. Rehabil Psychol 2010;55:272-85.

[33] Akaike H. A new look at the statistical model identification. IEEE Trans Automatic Control 1974;19(6):716-23.

[34] Albert N, Bertelsen M, Thorup A, Petersen L, Jeppesen P, Le Quack P, et al. Predictors of recovery from psychosis analyses of clinical and social factors associated with recovery among patients with first-episode psychosis after 5 years. Schizophr Res 2011;125(2-3):257-66.

[35] Harding CM. An examination of complexities in the measurement of recovery in severe psychiatric disorders. In: Ancill R, editor. Schizophrenia: Exploring the spectrum of psychosis. New York: John Wiley \& Sons; 1994. p. 153-69.

[36] Álvarez-Jiménez M, Gleeson JF, Henry LP, Harrigan SM, Harris MG, Killackey E, et al. Road to full recovery: longitudinal relationship between symptomatic remission and psychosocial recovery in first-episode psychosis over 7.5 years. Psychol Med 2012;42(3):595-606.

[37] Harrow M, Jobe TH. Factors involved in outcome and recovery in schizophrenia patients not on antipsychotic medications: a 15-year multifollow-up study. J Nerv Mental Dis 2007;195(5):406-14.

[38] Wunderink L, Nieboer RM, Wiersma D, Sytema S, Nienhuis FJ. Recovery in remitted first-episode psychosis at 7 years of follow-up of an early dose reduction/discontinuation or maintenance treatment strategy: long-term follow-up of a 2-year randomized clinical trial. JAMA Psychiatry 2013;70 (9):913-20.

[39] Wils RS, Gotfredsen DR, Hjorthøj C, Austin SF, Albert N, Secher RG, et al. Antipsychotic medication and remission of psychotic symptoms 10years after a first-episode psychosis. Schizophr Res 2017;182:42-8.

[40] Gaebel W, Riesbeck M, Wölwer W, Klimke A, Eickhoff M, von Wilmsdorff M, et al. Predictors for symptom re-exacerbation after targeted stepwise drug discontinuation in first-episode schizophrenia. Schizophr Res 2016;170 (1):168-76. 
[41] Lysaker PH, Klion Reid E. Recovery, meaning-making, and severe mental illness. A comprehensive guide to metacognitive reflection and insight therapy. New York: Routledge; 2018.

[42] Meyer PS, Mueser KT. Resiliency in individuals with serious mental illness. In: Southwick SM, Brett T, Litz DC, Friedman MJ, editors. Resilience and mental health: Challenges across the lifespan. Cambridge: Cambridge University Press; 2011.

[43] Cohn MA, Fredrickson BL, Brown SL, Mikels JA, Conway AM. Happiness unpacked positive emotions increase life satisfaction by building resilience. Emotion (Washington, DC) 2009;9(3):361-8. 